EPJ Web of Conferences 16, 06007 (2011)

DOI: $10.1051 /$ epjconf/20111606007

(C) Owned by the authors, published by EDP Sciences, 2011

\title{
Mid-infrared followup of cold brown dwarfs: Diversity in age, mass and metallicity
}

\author{
S.K. Leggett ${ }^{1, a}$, B. Burningham ${ }^{2}$, D. Saumon ${ }^{3}$, M.S. Marley ${ }^{4}$, \\ S.J. Warren ${ }^{5}$, H.R.A. Jones ${ }^{2}$, D.J. Pinfield ${ }^{2}$ and R.L. Smart ${ }^{6}$ \\ ${ }^{1}$ Gemini Observatory, 670 N. A'ohoku Place, Hilo, HI 96720, USA \\ ${ }^{2}$ Centre for Astrophysics Research, University of Hertfordshire, Hatfield AL10 9AB, UK \\ ${ }^{3}$ Los Alamos National Laboratory, PO Box 1663, MS F663, Los Alamos, NM 87545, USA \\ ${ }^{4}$ NASA Ames Research Center, Mail Stop 245-3, Moffett Field, CA 94035, USA \\ ${ }^{5}$ Imperial College London, Blackett Laboratory, Prince Consort Road, London SW7 2AZ, UK \\ 6 INAF/Osservatrio Astronomico di Torino, Strada Osservatrio 20, 10025 Pino Torinese, Italy
}

\begin{abstract}
We use Spitzer IRAC 3.6-8.0 $\mu$ m photometry of late-type T dwarfs to investigate various trends which can aid the planning and interpretation of infrared (IR) surveys for the coldest $\mathrm{T}$ or $\mathrm{Y}$ dwarfs. Brown dwarfs with effective temperature $\left(T_{\text {eff }}\right)<700 \mathrm{~K}$ emit $>50 \%$ of their flux at $\lambda>3 \mu \mathrm{m}$, and the ratio of the midIR to the near-IR flux becomes very sensitive to $T_{\text {eff }}$. The color $H-[4.5]$ is a good indicator of $T_{\text {eff }}$ with a weak dependence on metallicity $([\mathrm{m} / \mathrm{H}])$ and gravity $(g)$ while $H-K$ and $[4.5]-[5.8]$ are sensitive to $[\mathrm{m} / \mathrm{H}]$ and $g$. Thus $T_{\text {eff }}$ and $g$ can be constrained and mass and age can then be determined from evolutionary models. There are 12 dwarfs known with $H-$ [4.5] $>3.0$ and $500 \lesssim T_{\text {eff }} \mathrm{K} \lesssim 800$, which we examine in detail. The ages of these dwarfs range from very young $(0.1-1.0 \mathrm{Gyr})$ to old $(3-12 \mathrm{Gyr})$. The mass range is possibly as low as $5 \mathrm{M}_{\text {Jup }}$ to $70 \mathrm{M}_{\text {Jup }}$, and $[\mathrm{m} / \mathrm{H}]$ also spans a large range of $\sim-0.3$ to $\sim+0.3$. The T8-T9 dwarfs found so far in the UKIRT IR Deep Sky Survey are unexpectedly young and low-mass. Extensions to the warm Spitzer and WISE space missions are needed to obtain mid-IR data for cold brown dwarfs, and to discover more of these rare objects.
\end{abstract}

\section{INTRODUCTION}

The last decade has seen a remarkable increase in our knowledge of the bottom of the main-sequence and of the low-mass stellar and sub-stellar (brown dwarf) population of the solar neighbourhood. Two new classes have been added to the spectral type sequence following $\mathrm{M}$ : $\mathrm{L}$ and $\mathrm{T}$. $\mathrm{T}$ dwarfs with effective temperatures $\left(T_{\text {eff }}\right)$ as low as $\sim 500 \mathrm{~K}$ are now known (Warren et al. 2007; Burningham et al. 2008; Delorme et al. 2008; Leggett et al. 2009) and we are truly finding objects that provide the link between the low-mass stars and the giant planets.

As $T_{\text {eff }}$ decreases, brown dwarfs emit significant flux in the mid-infrared(IR) (e.g. Burrows et al. 2003; Leggett et al. 2009). Figure 1 demonstrates the rapidly increasing importance of this region for late-type T dwarfs; for dwarfs cooler than $700 \mathrm{~K}$ more than half the flux is emitted at wavelengths longer than $3 \mu \mathrm{m}$. Here we investigates photometric trends seen in very late-type dwarfs at mid-IR wavelengths, with the expectation that these longer wavelengths will be crucial for both the discovery and the understanding of the coolest T-type dwarfs, and even more so for the proposed cooler Y-type dwarfs. Our group was awarded time on the Spitzer Space Telescope (Werner et al. 2004) to obtain IRAC (Fazio et al. 2004) photometry of late-type T dwarfs found in the Large Area Survey (LAS) component of the UKIRT IR Deep Sky Survey (UKIDSS; Lawrence et al. 2007). Identification and classification of the LAS T dwarfs is described by Pinfield et al. (2008)

\footnotetext{
ae-mail: sleggett@gemini.edu
}

This is an Open Access article distributed under the terms of the Creative Commons Attribution-Noncommercial License 3.0, which permits unrestricted use, distribution, and reproduction in any noncommercial medium, provided the original work is properly cited. 


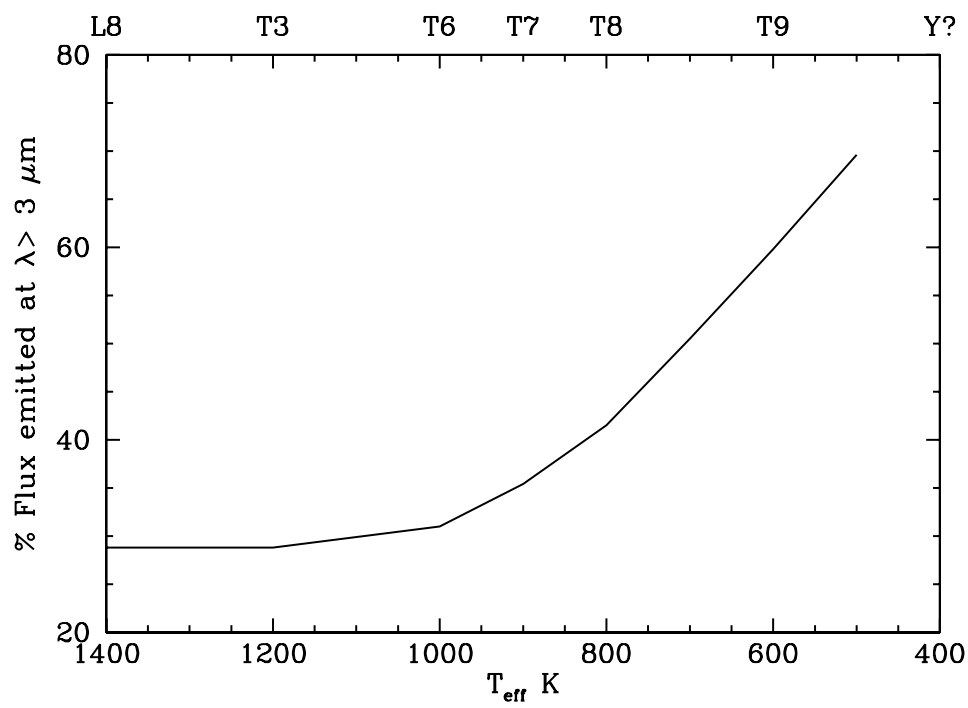

Figure 1. Percentage of total flux emitted at $\lambda>3 \mu \mathrm{m}$, as a function of $T_{\text {eff }}$, calculated from solar metallicity $\log g=5.0$ models which include vertical gas transport. Cloudy models were used for $T_{\text {eff }}>1000 \mathrm{~K}$ and cloudfree for cooler temperatures.

\section{THE SAMPLE}

Figure 1 demonstrates the rapidly increasing ratio of the mid-IR flux to the near-IR flux for late-type $\mathrm{T}$ dwarfs. This means that a large wavelength baseline near-IR to mid-IR color should be a good indicator of $T_{\text {eff }}$, and the $H-$ [4.5] color has been shown to be optimum (Warren et al. 2007; Leggett et al. 2009; Stephens et al. 2009). Leggett et al. (2009) show that expected variations in gravity ( $g$ ) and metallicity $([\mathrm{m} / \mathrm{H}])$, and in the gas transport efficiency, each impact $H-[4.5]$ by $\sim 0.2$ magnitudes. This is small given the large degree of sensitivity to $T_{\text {eff }}: \Delta(H-[4.5]) \approx 0.7$ magnitudes for $\Delta T_{\text {eff }}=100 \mathrm{~K}$ at $T_{\text {eff }}=600 \mathrm{~K}$ for example.

We adopt $H-$ [4.5] as a primary reference color and have selected T dwarfs with IRAC photometry that have $H_{\text {MKO }}-[4.5]>3.0$, which our models imply have $T_{\text {eff }} \lesssim 800 \mathrm{~K}$. Including the LAS T dwarfs with new IRAC photometry, $12 \mathrm{~T}$ dwarfs fall into this group. The dwarfs are listed in Table 1 together with some of their fundamental properties. All dwarfs with IRAC photometry that are T7.5 or later are included in this sample, except for the metal-rich T7.5 dwarf 2MASS J1217-03 (Saumon et al. 2007). Its exclusion is due to the (small) dependency of $H-[4.5]$ on $[\mathrm{m} / \mathrm{H}]$. Similarly, the relatively early-type very metal-poor dwarfs 2MASS J0937+29 (T6p) and 2MASS J1237+65 (T6.5e) have been included in our color-selected sample.

\section{TEMPERATURE, METALLICITY AND GRAVITY}

\subsection{Photometric Indicators}

Figure 2 shows a selection of color-magnitude diagrams for our sample, and Figure 3 color-color diagrams. Sequences are shown calculated by our cloud-free models which include chemical mixing by gas transport, for a range of $g$ and $[\mathrm{m} / \mathrm{H}]$. Although not perfect, the relative location of the objects in the plots is in agreement with our models. For example, metal-poor dwarfs are bright in Figure 2, and blue in $H-K$ and [4.5] - [5.8] in Figure 3. The values of $T_{\text {eff }}$ indicated for the sample in Figures 2 and 3 are consistent with other estimates (Table 1). 
Research, Science and Technology of Brown Dwarfs and Exoplanets

Table 1. T Dwarf Sample with $H_{\mathrm{MKO}}-[4.5]>3$

\begin{tabular}{|c|c|c|c|c|c|c|c|c|c|}
\hline \multirow[t]{2}{*}{ Name } & \multicolumn{2}{|c|}{ Spec. } & \multicolumn{4}{|c|}{ Physical Parameters } & \multicolumn{3}{|c|}{ References $^{\mathrm{a}}$} \\
\hline & Type & $T_{\text {eff }} \mathrm{K}$ & $\log g$ & {$[\mathrm{~m} / \mathrm{H}]$} & $\mathrm{M}_{\text {Jup }}$ & Age Gyr & Disc. & $\pi$ & Param. \\
\hline MASS J0937347+293142 & T6p & $925-975$ & $5.2-5.5$ & -0.3 & $45-69$ & $3-10$ & 1 & 2 & 3 \\
\hline 2MASS J12373919+6526148 & T6.5e & $800-850$ & $\sim 5.0$ & -0.2 & $20-40$ & $1-4$ & 4 & 2 & 5 \\
\hline 2MASS J11145133-2618235 & $\mathrm{T} 7.5$ & $725-775$ & $5.0-5.3$ & -0.3 & $30-50$ & $3-8$ & 6 & & 7 \\
\hline Gl 570D & $\mathrm{T} 7.5$ & $800-820$ & 5.1 & 0.00 & $31-47$ & $2-5$ & 8 & & 10 \\
\hline HD 3651B & $\mathrm{T} 7.5$ & $780-840$ & 5.3 & +0.15 & $40-72$ & $3-12$ & 11 & & 12 \\
\hline 2MASS J0415195-093506 & $\mathrm{T} 8$ & $725-775$ & $5.0-5.4$ & $\gtrsim 0$ & $33-58$ & $3-10$ & 1 & & 13 \\
\hline 2MASS J09393548-2448279b & $\mathrm{T} 8$ & $500-700$ & $5.0-5.3$ & -0.3 & $20-40$ & $2-10$ & 6 & & 14 \\
\hline ULAS J101721.40+011817.9c & T8p & $750-850$ & $4.0-4.5$ & $\gtrsim 0$ & $8-12$ & $0.1-0.4$ & 15 & & 16 \\
\hline ULAS J123828.51+095351.3 & T8.5 & $575-625$ & $4.0-4.5$ & $\gtrsim 0$ & $6-10$ & $0.2-1.0$ & 15 & & 16 \\
\hline Wolf $940 B^{d}$ & T8.5 & $545-595$ & $4.8-5.0$ & 0 to +0.3 & $20-32$ & $3.5-6$ & 17 & & 17 \\
\hline ULAS J003402.77-005206.7 & T9 & $550-600$ & 4.5 & $\gtrsim 0$ & $13-20$ & $1-2$ & 18 & 19 & 19 \\
\hline ULAS J133553.45+113005.2 & T9 & $500-550$ & $4.0-4.5$ & $\gtrsim 0$ & $5-20$ & $0.1-2$ & 15 & 20 & 14 \\
\hline
\end{tabular}

${ }^{\text {a }}$ Discovery, trigonometric parallax and parameter references are:

(1) Burgasser et al. (2002) (2) Vrba et al. (2004) (3) Geballe et al. (2009) (4) Burgasser et al. (1999)

(5) Liebert \& Burgasser (2007) (6) Tinney et al. (2005) (7) Leggett et al. (2007) (8) Burgasser et al. (2000)

(9) ESA (1997) (10) Saumon et al. (2006) (11) Mugrauer et al. (2006) (12) Liu et al. (2007) (13) Saumon et al. (2007) (14) Leggett et al. (2009) (15) Burningham et al. (2008) (16) This work (17) Burningham et al. (2009) (18) Warren et al. (2007) (19) Smart et al. in prep. (20) Smart et al. (2009) priv. comm.

${ }^{\mathrm{b}}$ Likely binary; a pair of $600 \mathrm{~K}$ dwarfs, or a $500 \mathrm{~K}$ and a $700 \mathrm{~K}$ brown dwarf pair (Burgasser et al. 2008; Leggett et al. 2009).

${ }^{c}$ If this spectrally peculiar object is a binary then $g$, mass and age, could be larger $-\log g=4.5-5.0$, masses $\sim 20 \mathrm{M}_{\text {Jup }}$, age $\sim 1 \mathrm{Gyr}$.

${ }^{\mathrm{d}}$ Burningham et al. (2009) give $[\mathrm{Fe} / \mathrm{H}]=-0.06 \pm 0.20$ based on the $V-K$ color of Wolf $940 \mathrm{~A}$; the photometric analysis presented here implies $[\mathrm{m} / \mathrm{H}]$ between $=0.0$ and +0.3 for the system.

2MASS $1237+65$ has very strong $\mathrm{H} \alpha$ emission. Liebert \& Burgasser (2007) discount the possibility that it is young and low- $g$, which would also be inconsistent with our analysis. They suggest that the object may be a close double system, and if the companion fills the Roche lobe then it must be cooler than $650 \mathrm{~K}$. As no significant [4.5] excess is seen in Figure 2, our models imply that any companion would have $T_{\text {eff }}<500 \mathrm{~K}$. The red $H-$ [4.5] for the dwarf is due to its low metallicity and not to the detected presence of a cool companion.

Figure 3 suggest that ULAS $1017+01$ and ULAS $1238+09$ are low gravity possibly metal-rich objects, similar to the other LAS very late-type T dwarfs ULAS 0034-07 and ULAS 1335+11, but not as cool. This low gravity for ULAS $1017+01$ disagrees with the value determined by Burningham et al. (2008) based on near-IR data only and implies a very young age of $0.1-0.4$ Gyr. This dwarf is spectrally peculiar; if the object is an unresolved $900+700 \mathrm{~K}$ or $800+600 \mathrm{~K}$ binary, then our model colors indicate that $g$ could be higher and the age would be $\sim 1$ Gyr, more typical for a local disk dwarf.

Figures 2 and 3 allow us to constrain the metallicity of the Wolf 940 system: Burningham et al. (2009) give $[\mathrm{Fe} / \mathrm{H}]=-0.06 \pm 0.20$ based on the $V-K$ color of Wolf $940 \mathrm{~A}$ but the photometric analysis presented here implies that $[\mathrm{m} / \mathrm{H}]$ for the system is between 0 and +0.3 . We can exclude the possibility that Wolf $940 \mathrm{~B}$ is metal-poor.

\subsection{The Low-Mass LAS Dwarfs}

The 2MASS-selected dwarfs tend to be high- $g$ and/or low- $[\mathrm{m} / \mathrm{H}]$ because they are selected for blue $H-K$ color (Figures 2 and 3). However the tendency for the LAS objects to be low- $g$ (Table 1) is difficult to understand. Figure 3 shows that the $Y J H$ colors used to select the LAS dwarfs are not sensitive to $g$ or $[\mathrm{m} / \mathrm{H}]$. There should also be no $g /$ age bias introduced by a brightness selection, as field 

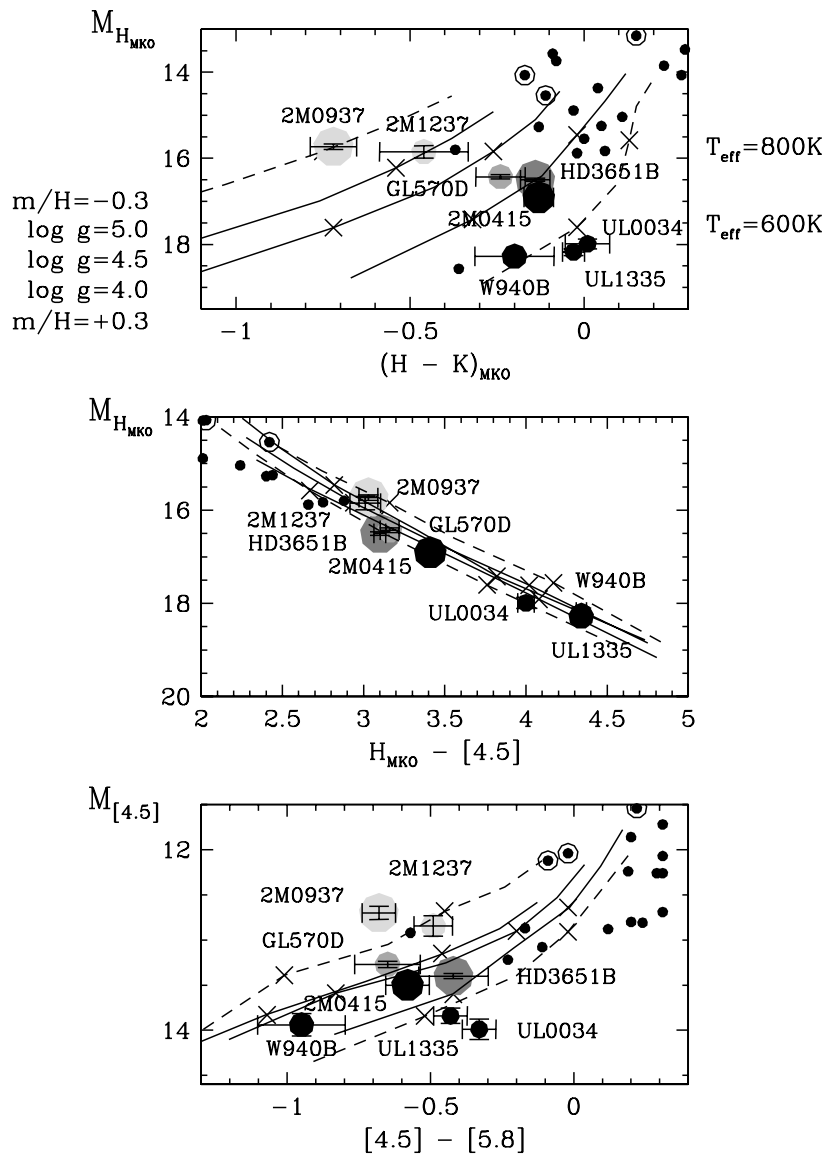

Figure 2. Color-magnitude plots for late $\mathrm{T}$ dwarfs. Small dots are a generic sample, known binaries are shown with ringed symbols. Larger symbols are the Table 1 dwarfs; largest to smallest circles represent $\log g \approx 5.4$, $\log g \approx 5.2, \log g \approx 5.0$ and $\log g \approx 4.3$. Dark to light grey circles are metal-rich, solar and metal-poor dwarfs; black are unknown $[\mathrm{m} / \mathrm{H}]$. Model sequences are shown with parameters indicated in the top panel. Crosses along the sequences indicate $T_{\text {eff }}=800 \mathrm{~K}$ and $600 \mathrm{~K}$.

brown dwarfs have an almost constant radius so that luminosity is effectively a function of $T_{\text {eff }}$ only (Saumon \& Marley 2008).

Simulations of the mass function (Burgasser 2004) show that the median field brown dwarf mass (and hence $g$ ) decreases to lower $T_{\text {eff }}$ because lower mass brown dwarfs start off cooler than higher mass brown dwarfs. However the simulations indicate that, for a flat mass function (e.g. Metchev et al. 2008, Pinfield et al. 2008) and constant birth-rate, at $T_{\text {eff }} \approx 600 \mathrm{~K}$ the median mass would be $30-40 \mathrm{M}_{\text {Jup }}$, age $>6 \mathrm{Gyr}$ and $\log g \approx 5$. The LAS T8-T9 dwarfs however appear to be generally younger than 2 Gyr and less massive than $20 \mathrm{M}_{\mathrm{Jup}}$. This puzzle will be re-examined when the sample of cold LAS brown dwarfs is larger.

\section{CONCLUSIONS}

The wavelength region beyond $3 \mu \mathrm{m}$ makes up most of the emitted flux for dwarfs cooler than $700 \mathrm{~K}$ and is crucial for analysis of their photospheres. This region is extremely challenging from the ground and extensions to the warm Spitzer and WISE missions are desirable. 
Research, Science and Technology of Brown Dwarfs and Exoplanets

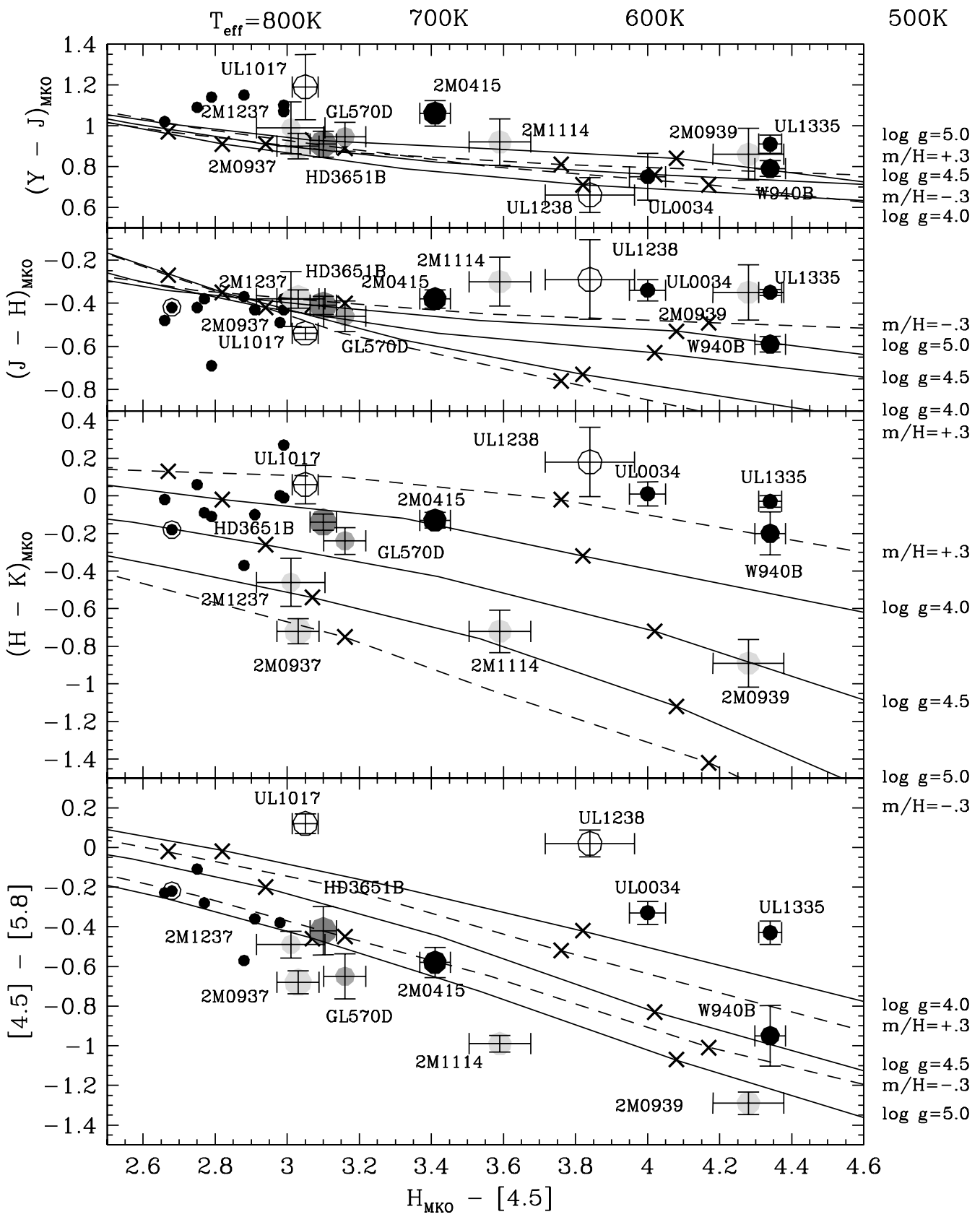

Figure 3. $H-$ [4.5] against $Y-J, J-H, H-K$ and [4.5] - [5.8]. Symbols are as in Figure 2 with open circles as unknown $g$. Model sequences are shown with parameters as indicated on the right axes. The $T_{\text {eff }}$ values for the $\log g=4.5[\mathrm{~m} / \mathrm{H}]=0$ model are indicated on the top axis, and crosses along the sequences indicate the $800 \mathrm{~K}$ and $600 \mathrm{~K}$ points for each model set.

The long-baseline color $H-$ [4.5] provides a good indicator of $T_{\text {eff }} H-K$ and [4.5] - [5.8] are sensitive to metallicity and gravity. As more data are obtained and the models improve it may be possible to separate these effects for independent determinations of these parameters. 
The photometry suggests that four of the five ULAS T8-T9 dwarfs are younger than 2 Gyr and less massive than $20 \mathrm{M}_{\mathrm{Jup}}$. This trend is not expected and not currently understood, we will revisit this as the UKIDSS survey continues and the sample size increases.

\section{References}

[1] Burningham B. et al. 2008, MNRAS 391320

[2] Burningham B. et al. 2009, MNRAS 3951237

[3] Burgasser A. J. et al. 1999 ApJ 522 L65

[4] Burgasser A. J. et al. 2000 ApJ 531 L57

[5] Burgasser A. J. et al. 2002 ApJ 564421

[6] Burgasser A. J. 2004 ApJS 155191

[7] Burgasser A. J. et al. 2008, ApJ 689 L53

[8] Burrows A., Sudarsky D., and Lunine J. I. 2003 ApJ 596587

[9] Delorme P. et al. 2008 A\&A 482961

[10] ESA 1997 The Hipparcos and Tycho Catalogues (ESA SP-1200, Noordwijk : ESA)

[11] Fazio, G. et al. 2004 ApJS 15410

[12] Geballe T. R. et al. 2009 ApJ 695844

[13] Lawrence A. et al. 2007 MNRAS 3791599

[14] Leggett S. K. et al. 2007 ApJ 667537

[15] Leggett S. K. et al. 2009 ApJ 6951517

[16] Liebert J. \& Burgasser A. J. 2007 ApJ 655522

[17] Liu M. C., Leggett S. K. \& Chiu K. 2007 ApJ 6601507

[18] Metchev S. A. et al. 2008 ApJ 6761281

[19] Mugrauer M. et al. 2006 MNRAS 373 L31

[20] Pinfield D J 2008 MNRAS 390304

[21] Saumon D. et al. 2006 ApJ 647552

[22] Saumon D. et al. 2007 ApJ 6621245

[23] Saumon D. and Marley M. S. 2008 ApJ 6891327

[24] Stephens D. S. et al. 2009, ApJ, 702154

[25] Tinney C. G. et al. 2005 AJ 1302326

[26] Vrba F. J. et al. 2004 AJ 1272948

[27] Warren S. J. et al. 2007 MNRAS 3811400

[28] Werner M. W. et al. 2004 ApJS 1541 